\title{
Diversidad genética de dos poblaciones del caracol Strombus gigas (Gastropoda: Strombidae) en Yucatán, México, con microsatélite
}

\author{
Roberto Zamora-Bustillos ${ }^{1,2}$, Rossanna Rodríguez-Canul ${ }^{2}$, Francisco J. García de León ${ }^{3}$ \\ \& Jorge Tello Cetina ${ }^{4}$ \\ 1. División de Posgrado e Investigación, Instituto Tecnológico de Conkal, Km. 16.3 antigua carretera Mérida-Motul, C.P \\ 97345, Conkal, Yucatán, México; roberto.zamora@itconkal.edu.mx \\ 2. Departamento de Recursos del Mar, CINVESTAV-IPN, Unidad Mérida, Km 6 Antigua carretera a Progreso, C.P \\ 97310, Apartado Postal 73, Mérida, Yucatán, México; rossana@mda.cinvestav.mx \\ 3. Laboratorio de Genética para la Conservación, Centro de Investigaciones Biológicas del Noroeste, S.C. Mar Bermejo \\ No. 195, Col. Playa Palo de Santa Rita. Apdo. Postal 128; La Paz, BCS 23090, México; fgarciadl@cibnor.mx \\ 4. División de Posgrado e Investigación, Instituto Tecnológico de Mérida, Av. Tecnológico s/n Apartado postal 9-11, \\ Mérida Yucatán, México; jorgegigas1@gmail.com
}

\author{
Recibido 13-IX-2010. C Corregido 02-II-2011. Aceptado 01-III-2011.
}

\begin{abstract}
Genetic diversity in two populations of the snail Strombus gigas (Gastropoda: Strombidae) from Yucatan, Mexico, using microsatellite. The pink conch Strombus gigas is an important fisheries resource in the Caribbean region, including the Yucatán Peninsula. We analyzed the genetic diversity and genetic structure of two populations (Alacranes Reef and Chinchorro Bank) with the use of five microsatellite molecular markers. The results indicate that the two populations are in the same rank of genetic diversity $(\mathrm{He})$, from 0.613 to 0.692. Significant deviation from H-WE was observed in the both populations due to deficit to heterozygotes, this was attributed to inbreeding as a consequence of over-fishing; nevertheless, other possible causes considered are mixing of individuals from two or more populations, and the existence of null alleles. Levels of genetic differentiation indicated the existence of a single homogenous population in the Yucatan Peninsula ( $\mathrm{F}_{\mathrm{ST}}$ de 0.003 , $\mathrm{p}=0.49$ ), which fits with highest levels of gene flow is significant (2.3 individuals) between both populations. Results from this study support the hypothesis that $S$. gigas is part of a single panmictic population in the Yucatan Peninsula; therefore, this fishery resource should be regulated the same way for both areas. Rev. Biol. Trop. 59 (3): 1127-1134. Epub 2011 September 01.
\end{abstract}

Key words: Strombus gigas, pink conch, microsatellite, genetic diversity, gene flow.

El manejo sustentable de cualquier especie requiere el conocimiento de los niveles de diferenciación genética de las subunidades poblacionales, dado que su desconocimiento dentro del ámbito geográfico repercuten en la explotabilidad y sustentabilidad del recurso (Han et al. 2008). Bajo la óptica de la conservación a largo plazo, la utilización de la genética molecular para estimar el grado de aislamiento reproductivo entre poblaciones, ha permitido reconocer diferencias intraespecíficas que son heredables y evolutivamente relevantes (Grant et al. 1999). Por ello las evaluaciones de los niveles de diversidad genética de las poblaciones toman especial importancia cuando la especie es económicamente importante, puesto que obliga a desarrollar mejores estrategias de manejo fundadas en el conocimiento de la identidad genética de las poblaciones.

La gestión de los recursos pesqueros de importancia y la determinación de su diversidad genética, con la finalidad de diferenciar stocks, es de gran utilidad para maximizar de manera sostenible el rendimiento de las capturas e identificar el impacto de actividades antrópicas en procesos ecológicos y evolutivos 
(Moritz 1994). Así también resulta importante reconocer que es a este nivel (stock discretos o demes) en donde se establecen las variaciones genéticas y la capacidad de la especie para responder a condiciones ambientales particulares, al incrementar su habilidad para soportar perturbaciones ocasionadas por la pesca o degradación del hábitat (Allison et al. 2003).

La consideración de utilizar estos argumentos en el estudio del caracol rosado $S$. gigas, gasterópodo marino de gran relevancia biológica y que representa un recurso biológico de gran importancia en el Caribe, reside en el hecho de que este organismo se encuentra sujeto a una sobreexplotación, que presumiblemente ha mermado drásticamente sus poblaciones naturales (Acosta 2006). El caracol rosado, debido a su prologado tiempo de metamorfosis, 28 días promedio en la columna de agua, tiene la capacidad de dispersarse a grandes distancias, lo cual conlleva a pensar en la posibilidad de que existan pequeñas diferencias genéticas significativas entre las poblaciones del molusco en regiones diferentes (Mitton et al. 1989). Son pocos los estudios genéticos realizados en esta especie, entre ellos destaca el concerniente a la determinación de la posible diferenciación genética de las poblaciones de este recurso pesquero en su zona de distribución en el CaribeAtlántico empleando isoenzimas (Mitton et al.1989, Tello et al. 2005). Estos autores no encontraron diferencias genéticas significativas en sus áreas de muestreo. Es conocido que la variación isoenzimática tiene limitaciones para detectar sutiles niveles de diferenciación genética (Liu \& Cordes 2004). Recientemente Zamora-Bustillos et al. (2007), reportaron ocho marcadores moleculares tipo microsatélites, de los cuales sugieren cinco altamente polimórficos para el estudio de la diversidad genética en las poblaciones de esta especie. Estos marcadores tienen la ventaja de ser altamente polimórficos, lo que permite detectar pequeñas diferencias genéticas (Powell et al. 1996).

El conocimiento de diversidad y estructura genética de $S$. gigas es importante para entender la biología de las poblaciones e inferir el impacto de las diferentes fuerzas evolutivas (mutación, deriva génica y sistemas de reproducción) que han originado, mantenido y hecho evolucionar el acervo genético de éstas. Dicha información es básica para predecir el potencial de evolución y el riesgo de extinción ante la amenaza de su continua sobreexplotación.

Mediante el uso de una batería de cinco loci microsatélites, se demostró la existencia de una sola población homogénea en la Península de Yucatán y estudió su nivel de diversidad genética. Para planes de conservación, esta información es útil en la aplicación de medidas regulatorias, para aconsejar a los administradores del recurso pesquero, considerar la aplicación de la veda permanente para todas las zonas de captura de $S$. gigas en la península de Yucatán.

\section{MATERIALES Y MÉTODOS}

Recolecta de organismos: Un total de 32 caracoles se recolectaron en el Parque Nacional Arrecife Alacranes (A.A) (21 ${ }^{\circ} 26^{\prime} 40^{\prime}$ ' $\mathrm{N}-86^{\circ} 47^{\prime} 64^{\prime \prime}$ W) al norte de la península de Yucatán y 40 caracoles en la Reserva Ecológica Banco Chinchorro (B.CH) (1848'44.2"

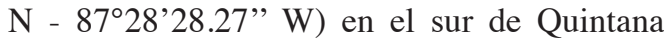
Roo. Al pie de cada caracol se le disectó aproximadamente $1 \mathrm{gr}$ de tejido y se conservó en etanol absoluto.

Extracción del ADN y amplificación por PCR: Mediante el kit de purificación $\left(\right.$ Promega ${ }^{\circledR}$ ) se realizó la extracción del ADN genómico de las muestras de tejido del caracol $S$. gigas; se analizaron cinco loci microsatélites (Sgig-1, Sgig-2, Sgig-3, Sgig-4 y Sgig-5 de acuerdo a Zamora-Bustillos et al. (2007). Los productos de la amplificación fueron separados con un equipo de electroforesis vertical SequiGenII GT ${ }^{\circledR}$ Nucleic Acid Electrophoresis Cell, (BIO-RAD) con solución amortiguadora $1 \mathrm{X}$ TBE, en geles desnaturalizantes de poliacrilamida al 6-10\% y urea 7M. Los alelos fueron separados entre 1800-2000 V empleando una fuente poder Power Pac 3000 (BIO-RAD) por dos horas aproximadamente. Para identificar el tamaño de los alelos se utilizó un patrón de 
peso molecular de 100pb (Promega ${ }^{\circledR}$ ). La tinción y revelado de los geles de poliacrilamida se realizó con nitrato de plata siguiendo el protocolo de Bassam \& Anollés (1993).

La lectura de las bandas se realizó con un transluminador de luz blanca tomando como guía el peso molecular incluido en los geles. Una vez hecha la lectura de las bandas, cada gel fue documentado con una cámara digital (Nikon Coolpix 7600).

Los genotipos obtenidos con los 5 loci en las dos poblaciones fueron analizados con el programa Micro-checker (Van Oosterhout et al. 2004), con la finalidad verificar la presencia de alelos nulos. Para estimar la diversidad genética se utilizaron diversas medidas tales como: número de alelos por locus $(\mathrm{Na})$, heterocigosidad esperada $(\mathrm{He})$ y heterocigosidad observada (Ho) con el programas Genetix versión 4.05 (Belkhir et al. 2004). El índice de Contenido Polimórfico $\left(P_{\mathrm{IC}}\right)$ se calculó con el programa CERVUS, versión 2.0 (Marshall et al. 1998), el cual provee un estimado del poder discriminatorio de cada locus tomando en cuenta, no sólo el número de alelos que son expresados, sino también la frecuencia relativa de estos alelos y su valor medio. Para probar si las poblaciones de $S$. gigas se encontraban en equilibrio de Hardy-Weinberg (H-WE) se utilizaron pruebas exactas de Fisher (5.000 demorizaciones, 100 batches y 1.000 iteraciones por batch), utilizando el programa Genepop versión 4.0.10 (Raymond \& Rousset 1995).

Para evaluar la diferenciación genética entre las dos poblaciones se realizó la prueba de diferenciación genética mediante el análisis de $\mathrm{F}_{\mathrm{ST}}$ pareados como se describe en el programa Arlequin ver 3.5 (Excoffier et al. 2005). Los valores de probabilidad $(\mathrm{p}<0.05)$ para estas comparaciones se obtuvieron mediante la proporción de 1000 permutaciones (Excoffier et al.2005). Una estimación del flujo génico entre ambas poblaciones se obtuvo con el método de alelos privados (Barton \& Slatkin 1986, Slatkin 1994) con el programa Genepop versión 4.0.1 de Raymond \& Rousset (1995).

\section{RESULTADOS}

La presencia de alelos nulos solo se detectó en el locus Sgig-5 en la población de A.A con frecuencia 0.40 , mientras que para el mismo locus para la muestra B.CH no se encontró frecuencias significativas de alelos nulos, al igual que con el resto de los loci para ambas muestras (Sgig-1, Sgig-2, Sgig-3 y Sgig-4) (datos no mostrados).

En el Cuadro 1 se muestra los índices de diversidad genética por locus, de cada población. El tamaño de muestra promedio estuvo alrededor de 35 individuos en todos los loci. El número mínimo y máximo de alelos por locus $\left(N_{a}\right)$ osciló entre cuatro y seis alelos para los loci Sgig-2, Sgig-3, Sgig-4 y Sgig-5, respectivamente. La muestra que presentó el mayor número de alelos por locus fue A.A con seis alelos para los loci Sgig-4 y Sgig-5, respectivamente. La media de la heterocigocidad observada Ho por locus en ambas poblaciones fue inferior al 0.50 en tres loci: Sgig-1 (0.422), Sgig-3 (0.475), Sgig-4 (0.27) y Sgig-5 (0.1), mientras que solo el locus Sgig-2 presentaron valores mayor al $50 \%$ en ambas poblaciones (0.70 y 0.56 , respectivamente). Los resultados de heterocigocidad esperada $\mathrm{He}$ para cada locus en ambas muestras fue mayor a 0.50 en la mayoría de los loci, a excepción del locus Sgig-4 que fue de 0.27. En todos los loci se observaron diferencias entre $\mathrm{He}$ y $\mathrm{Ho}$. Estas diferencias fueron provocadas por el déficit de heterocigotos que presentan la mayoría de los loci dentro de cada muestra. Sin embargo, con la prueba exacta de Fisher $\left(P_{H W}\right)$, se comprobó que solo dos loci (Sgig-1 y Sgig-5) se desviaron del equilibrio de H-WE, después de la corrección secuencial de Bonferroni (Rice 1989). La menor diversidad genética mediante el estimador $P_{\text {IC }}$ se encontró en el locus Sgig-4 y Sgig-5 con valor promedio de 0.432 , mientras el locus que presentó el mayor promedio fue Sgig-1 con 0.608 .

A nivel global, la diversidad genética promedio registrada mediante cinco loci 
CUADRO 1

Variación genética de cinco loci microsatélites en dos poblaciones de S. gigas en la costa de Yucatán

TABLE 1

Genetic variation of five microsatellite loci in two populations of S. gigas on the coast of Yucatan

\begin{tabular}{|c|c|c|c|c|c|c|c|}
\hline Locus & $N$ & $\mathrm{Na}$ & $\mathrm{He}$ & Ho & $P_{\text {IC }}$ & $P_{H W}$ & Población \\
\hline \multirow[t]{2}{*}{ Sgig-1 } & 31 & 5 & 0.597 & 0.419 & 0.552 & $0.000 * *$ & A.A \\
\hline & 40 & 5 & 0.790 & 0.425 & 0.664 & $0.000 * *$ & B.CH \\
\hline Promedio & 35.5 & 5 & 0.693 & 0.422 & 0.608 & & \\
\hline \multirow[t]{2}{*}{ Sgig-2 } & 30 & 4 & 0.645 & 0.766 & 0.576 & $0.904{ }^{\mathrm{NS}}$ & A.A \\
\hline & 40 & 4 & 0.584 & 0.763 & 0.757 & 0.998 NS & B.CH \\
\hline Promedio & 35 & 4 & 0.614 & 0.764 & 0.666 & & \\
\hline \multirow[t]{2}{*}{ Sgig-3 } & 32 & 4 & 0.600 & 0.500 & 0.550 & $0.028^{\mathrm{NS}}$ & A.A \\
\hline & 38 & 4 & 0.556 & 0.450 & 0.516 & $0.017^{\mathrm{NS}}$ & B.CH \\
\hline Promedio & 35 & 4 & 0.578 & 0.475 & 0.533 & & \\
\hline \multirow[t]{2}{*}{ Sgig-4 } & 31 & 6 & 0.364 & 0.290 & 0.350 & $0.079^{\mathrm{NS}}$ & A.A \\
\hline & 40 & 4 & 0.381 & 0.250 & 0.514 & $0.016^{\mathrm{NS}}$ & B.CH \\
\hline Promedio & 35.5 & 5 & 0.372 & 0.27 & 0.432 & & \\
\hline \multirow[t]{2}{*}{ Sgig-5 } & 31 & 6 & 0.861 & 0.125 & 0.350 & $0.000 * *$ & A.A \\
\hline & 40 & 4 & 0.848 & 0.075 & 0.514 & $0.000 * *$ & B.CH \\
\hline Promedio & 35.5 & 5 & 0.854 & 0.1 & 0.432 & & \\
\hline
\end{tabular}

Valores no significativos después de la aplicación de la corrección secuencial de Bonferroni $\alpha=0.05 / k=10, p<0.005$.

Número de individuos (N), Número de alelos por locus (Na), Heterocigocidad observada (Ho), Heterocigocidad esperada $(\mathrm{He})$, Contenido de la información polimórfica $\left(\mathrm{P}_{\mathrm{IC}}\right)$ y valor de probabilidad $\left(\mathrm{P}_{\mathrm{HW}}\right)$. Arrecife alacranes (A.A) y Banco Chinchorro (B. CH).

Number of individuals (N), Number of alleles per locus (Na), Observed heterozygosity (Ho), Expected heterozygosity (He), Polymorphic information content $\left(\mathrm{P}_{\mathrm{IC}}\right)$ and probability value $\left(\mathrm{P}_{\mathrm{HW}}\right)$. Alacranes Reef (A.A) and Chinchorro Bank (B. CH).

microsatélites en dos poblaciones de $S$. gigas, fue moderada (Cuadro 2). Los valores de $P_{\text {IC }}$ fueron mayores a 0.557 para ambas poblaciones, y la heterocigosidad esperada (He) mostró valores que oscilaron entre 0.613 y 0.632 , para
A.A y B.CH respectivamente; mientras que la heterocigosidad observada $(\mathrm{Ho})$ mostró valores que oscilaron entre $0.392-0.420$, para A.A y B.CH, respectivamente. Los valores de Fis mostraron indicios de endogamia. Además,

\section{CUADRO 2}

Análisis multilocus para la desviación a ley de Hardy-Weinberg $\left(\mathrm{P}_{\mathrm{HW}}\right)$

TABLE 2

Multilocus analysis for the Hardy-Weinberg law $\left(\mathrm{P}_{\mathrm{HW}}\right)$

$\begin{array}{cccccc}\text { Población } & H e & H o & P_{\mathrm{IC}} & \text { Fis } & P_{H W} \\ \text { A.A } & 0.613 & 0.420 & 0.576 & 0.336^{* *} & 0.000^{* *} \\ \text { B. CH } & 0.632 & 0.392 & 0.557 & 0.396^{* *} & 0.000^{* *}\end{array}$

**p<0.01 (nivel de significancia después de la corrección secuencial de Bonferroni).

Heterocigocidad observada (Ho), Heterocigocidad esperada $(\mathrm{He})$, Contenido de la información polimórfica $\left(\mathrm{P}_{\mathrm{IC}}\right)$ y coeficiente de endogamia (Fis). Arrecife alacranes (A.A) y Banco Chinchorro (B. CH).

Observed heterozygosity $(\mathrm{Ho})$, Expected heterozygosity $(\mathrm{He})$, Polymorphic information content $\left(\mathrm{P}_{\mathrm{IC}}\right)$ and inbreeding coefficient (Fis). Alacranes Reef (A.A) and Chinchorro Bank (B. CH). 
se confirmó la falta de divergencia poblacional mediante el análisis $\mathrm{F}_{\mathrm{ST}}$ entre pares de poblaciones, en donde se obtuvo un valor de $\mathrm{F}_{\mathrm{ST}}$ de 0.003 no significativo $(\mathrm{p}=0.499)$. El número de migrantes $(\mathrm{Nm})$ estimado para ambas poblaciones fue de 2.3 individuos por generación, lo cual asegura que éste número es suficiente para homogenizar genéticamente a estas dos poblaciones.

\section{DISCUSIÓN}

Los estimadores multilocus de $\mathrm{He}$ y $P_{\mathrm{IC}}$ mostraron valores medios y bajos de diversidad genética moderada en las 2 muestras analizadas, estos valores de $\mathrm{He}$ y $P_{\mathrm{IC}}$ son similares a los observados en otros invertebrados marinos, en los que se han utilizado los microsatélites. El déficit de heterocigotos es una característica comúnmente observada en moluscos marinos (Crassostrea gigas y Buccinum undatum) (Hedgecock et al. 2004, Weetman et al. 2005, respectivamente) y otros moluscos bivalvos (Crassostrea virginica, Venerupis pullastra y Macoma balthica) (Reece et al. 2004, Becquet et al. 2009, Pereira et al. 2009, respectivamente). Para el bivalvo Pinctada máxima, Lind et al. (2007) reportaron valores promedio de $H e=0.448$ y $P_{I C}=0.562$, ellos observaron dos loci que se apartaron del equilibrio de H-WE, mostrando un déficit de heterocigotos. En el gasterópodo marino Melongena corona, Hayes \& Kart (2004), encontraron valores de $H e=0.624$ con significante deficiencia de heterocigotos, argumentado la presencia de alelos nulos como la causa principal. En el caso de las dos muestras de $S$. gigas analizadas en este estudio, los valores diversidad genética (He) observados fueron moderado a bajos, y también se apartan del H-WE. Sokolov et al. (2002) y Weetman et al. (2005) atribuyeron el déficit de heterocigotos observados en especies de gasterópodos marinos a la presencia de alelos nulos como la principal causa. También Becquet et al. (2009) analizando el bivalvo Macoma balthica encontraron que la mayoría de los loci se desviaron del H-WE y concluyen que se da la presencia de alelos nulos más que factores relacionados con la panmixia o errores de genotipificación. En este estudio solo se detectó la presencia de alelos nulos en el locus Sgig-5 en ambas muestras. Sin embargo, no hay certeza que estos alelos nulos en este locus tengan un efecto significativo en el déficit de heterocigotos en ambas muestras.

La endogamia puede ser también un buen argumento para explicar las causas de deficiencia de heterocigotos a través de la reproducción de individuos emparentados (Addison \& Hart 2005). En especies de sexos separados como el caso de $S$. gigas, la endogamia puede verse favorecida debido a una reducción de sus abundancias a lo largo de su área de distribución, como consecuencia de la captura de caracoles adultos que son vulnerables y que corresponde a aquellos que migran a zonas de menor profundidad. En algunas áreas de distribución, las densidades de adultos de $S$. gigas son alarmantemente bajas debido ya sea, a la sobre-pesca o por la destrucción del hábitat; esta condición ocasiona que los adultos que habitan en un sitio en particular no sean suficientes para permitir el cambio de pareja en un ciclo reproductivo en un área reducida. Los individuos tienen dificultades para cambiar de pareja en un ciclo reproductivo en un área reducida, por lo que se ven obligados en la mayoría de las veces a reproducirse con los mismos organismos que se encuentran en el área. A este proceso se le conoce como efecto Allee o depensación (Møller \& Legendre 2001). El efecto Allee es un fenómeno común en especies de invertebrados marinos de poco movimiento como corales, esponjas, mejillones, ostras y caracoles (Gascoigne \& Lipcius 2004). De esto se considera, que el éxito reproductivo depende en gran medida de la densidad de los individuos y la distancia donde se encuentra el individuo del sexo opuesto para la cópula (Nielsen \& Kenchington 2001). En el caso de $S$. gigas, durante el periodo reproductivo abundan la hembras y se dificulta localizar a un macho para la cópula (Acosta 2006); este fenómeno puede explicar las desviaciones al H-WE observadas.

Otro mecanismo también altamente plausible para explicar el déficit de heterocigotos, se 
basa en el efecto Wahlund, el cual se refiere a la reducción de heterocigocidad en una población causada por una estructura subpoblacional o mezcla de poblaciones (Tripp-Valdéz et al. 2010). De igual forma, los organismos que producen gran número de gametos, tal como los gasterópodos marinos, tienen un mayor número de ciclos celulares, por lo tanto se incrementa la posibilidad de mutaciones a nivel de la replicación del ADN (Foltz 1986, Beckenbach 1994), se ha observado que las especies con espermatozoides planctónicos muestran alelos nulos y desviaciones al H-WE, en comparación con especies de menor frecuencia copulatoria, y menor producción de espermatozoides como los decápodos en el cual la presencia de alelos nulos es rara (Jensen \& Bentzen 2004). Un último mecanismo que podría explicar el déficit de heterocigotos se basa en la sobre explotación que directamente aumenta la deriva génica (Benzie \& Williams 1998), cabe señalar que los efectos de sobrepesca no solo afectan los sitios de pesca sobre los que inciden sino que también podrían causar un efecto a largo plazo sobre los patrones de reclutamiento larvario en sitios de pesca lejanos. Por tanto, se requieren estudios más detallados que impliquen un mayor número de loci microsatélites y una red de muestreo más amplia para discernir entre las explicaciones antes mencionadas.

El valor de $\mathrm{F}_{\mathrm{ST}}$ entre pares de poblaciones no fue significativo, lo cual indica que las muestras analizadas no se diferencian genéticamente y el flujo de genes entre las ellas es lo suficientemente grande como para homogenizar las dos poblaciones analizadas. La estimación del número de migrantes por generación $(\mathrm{Nm})$ entre las poblaciones fue de más de dos individuos, lo que se considera suficiente para prevenir los efectos de la estructuración genética en las poblaciones (Slatkin \& Barton 1989). Según Slatkin (1994), bajo el equilibrio de mutación y deriva se requiere más de un migrante $(N m>1)$ en promedio por generación para impedir la diferenciación genética por efecto de deriva génica.

Para muchas especies marinas con larvas pelágicas, la homogeneidad genética se demuestra a menudo sobre grandes regiones geográficas debido a la dispersión de las larvas por las corrientes marinas (Zi-niu et al. 2005). Frecuentemente las larvas de S. gigas son transportadas grandes distancias geográficas $(\approx 900 \mathrm{~km}$ ) durante las tres primeras semanas de vida, en el todo el Caribe y Golfo de México (Davis et al. 1993).

Con el análisis realizado se concluye que entre los dos sitios de captura del norte de la península de Yucatán y la costa Caribeña en el sur de la península de Yucatán no existen diferencias genéticamente significativas, por lo que se interpreta que los caracoles presentes en ambos sitios de muestro intercambian genes y forman una misma unidad panmíctica. Por lo tanto, estas dos áreas donde se distribuye $S$. gigas deben manejarse con los mismos planes de uso y conservación.

\section{AGRADECIMIENTOS}

Este estudio fue financiado por los servicios externos del Laboratorio de Inmunología y Biología Molecular del CINVESTAV IPN Unidad Mérida (Número de Referencia: A3329). A Luis A. Rodríguez Gil por su valiosa colaboración en la recolecta de las muestras.

\section{RESUMEN}

El caracol rosado $S$. gigas, es una especie de gran importancia pesquera en la región del Caribe que incluye la Península de Yucatán, en la cual, se analizó la diversidad y estructura genética de dos poblaciones (Arrecife Alacranes y Banco Chinchorro) mediante el uso de cinco marcadores moleculares del tipo microsatélites. Los resultados indican que las dos poblaciones analizadas se encuentran en el mismo rango de diversidad genética $(\mathrm{He})$ de 0.613 a 0.692 . En ambas poblaciones también se observó una desviación significativa al equilibrio $\mathrm{H}-\mathrm{WE}$, la cual fue atribuida a factores como la endogamia a consecuencia de una sobre-explotación pesquera. Sin embargo otra explicación posible es que se deba a una mezcla de individuos de dos o más poblaciones, y la existencia de alelos nulos. Los niveles de estructura genética indican la existencia de una sola población homogénea en la península de Yucatán $\left(\mathrm{F}_{\mathrm{ST}}\right.$ de $\left.0.003, \mathrm{p}=0.49\right)$ y el flujo genético fue significativo (2.3 individuos) entre las dos poblaciones. Los resultados de este estudio aceptan la hipótesis de que las poblaciones $S$. gigas forman parte de una sola población panmíctica en 
la Península de Yucatán, por lo tanto, el recurso pesquero debe regularse de igual manera en ambas regiones.

Palabras clave: Strombus gigas, caracol rosado, microsatélites, diversidad genética, flujo genético.

\section{REFERENCIAS}

Acosta, C.A. 2006. Impending trade suspensions of Caribbean Queen Conch under CITES: A case study on fishery impacts and potential for stock recovery. Fisheries 31: 601-606.

Addison, J.A. \& M.W. Hart. 2005. Spawning, copulation and inbreeding coefficients in marine invertebrates. Biol. Lett.1: 450-3.

Allison, G.W., S.D. Gaines, J. Lubchenco \& H.P. Possingham. 2003. Ensuring persistence of marine reserves: catastrophes require adopting an insurance factor. Ecol. Appl. 13: 8-24.

Barton, N.H. \& M. Slatkin. 1986. A quasi-equilibrium theory of the distribution of rare alleles in a subdivided population. Heredity 56: 409-415.

Bassam, B.J. \& G.C. Anollés. 1993. Silver staining of DNA in polyacrylamide gels. Appl. Biochem. Biotechnol. 42: 181-188.

Beckenbach, A.T. 1994. Mitochondrial haplotype frequencies in oysters: neutral alternatives to selection models, p. 188-198. In B. Golding (ed.). Non-neutral evolution: theories and molecular data. Chapman \& Hall, Nueva York, EEUU.

Becquet, V., I. Lanneluc, B.S. Bouhet \& P. García. 2009. Microsatellite markers for the Baltic clam, Macoma balthica (Linné, 1758), a key species concerned by changing southern limit, in exploited littoral ecosystems. Conserv. Genet. Resour. 1: 265-267.

Belkhir, K., P. Borsa, V. Chikhi, N. Raufaste \& F. Bonhomme. 2004. GENETIX 4.05, logiciel sous Windows TM pour la génétique des populations. Laboratoire Génome, Populations, Interactions, CNRS UMR 5000, Université de Montpellier II, Montpellier, Francia.

Benzie, J.A.H. \& S.T. Williams. 1998. Phylogenetic relationships among giant clam species (Mollusca: Tridacnidae) determined by protein electrophoresis. Mar. Biol. 132: 123-133.

Davis, M., C.A. Bolton \& A.W. Stoner. 1993. A comparison of larval development, growth, and shell morphology in three Caribbean Strombus species. Veliger 36: $236-244$.
Excoffier, L., G. Laval \& S. Schneider. 2005. Arlequin version 3.0: an integrated software package for population genetics data analysis. Evol. Bioinform. Online 1: 47-50.

Foltz, D.W. 1986. Segregation and linkage studies of allozyme loci in pair crosses of the oyster Crassostrea virginica. Biochem. Genet. 24: 941-956.

Gascoigne, J. \& R. Lipcius. 2004. Allee effects in marine systems. Mar. Ecol. Prog. Ser. 269: 49-59.

Grant, W.S., J.L. Garcia-Marin \& F.M. Utter. 1999. Defining population boundaries for fishery management, p. 27-72. In S. Mustafa (ed.). Genetics in sustainable fisheries management. Blackwell Scientific, Oxford, Inglaterra.

Han, Y.S., Y.L. Sun, Y.F. Liao, I.C. Liao, K.N. Shen, W.N. Tzeng. 2008. Temporal analysis of population genetic composition in the overexploited Japanese eel Anguilla japonica. Mar. Biol. 155: 613-621.

Hayes, K.A. \& S.A. Karl. 2004. Characterization of microsatellite markers from the gastropod genus Melongena corona. Mol. Ecol. Notes 4: 755-757.

Hedgecock, D., G. Li, S. Hubert, K. Bucklin \& V. Ribes. 2004. Widespread null alleles and poor cross-species amplification of microsatellite DNA loci cloned from the Pacific oyster, Crassostrea gigas. J. Shell. Res. 23: 379-385.

Jensen, P.C. \& P. Bentzen. 2004. Isolation and inheritance of microsatellite loci in the Dungeness crab (Brachyura: Cancridae: Cancer magister) Genome 47: 325-331.

Lind, C.E., B.S. Evans, J.J. Taylor \& D.R. Jerry. 2007. Population genetics of a marine bivalve, Pinctada maxima, throughout the indo-australian archipelago shows differentiation and decreased diversity at range limits. Mol. Ecol. 16: 5193-203.

Liu, Z.J. \& J.F. Cordes. 2004. DNA marker technologies and their applications in aquaculture genetics. Aquaculture 23: 1-37.

Marshall, T.C., J. Slate, L. Kruuk \& J.M. Pemberton. 1998. Statistical confidence for likelihood-based paternity inference in natural populations. Mol. Ecol. 7: 639-655.

Mitton, J.B., C.J. Berg \& K.S. Orr. 1989. Population structure, larval dispersal, and gene flow in the Queen Conch, Strombus gigas, of the Caribbean. Biol. Bull. 177: 356-362. 
Møller, A.P. \& S. Legendre. 2001. Allee effect, sexual selection and demographic stochasticity. Oikos 92: 27-34.

Moritz, C.1994. Defining evolutionary significant units for conservation. Trends Ecol. Evol. 9: 373-375.

Nielsen, E.E. \& E.E. Kenchington. 2001. A new approach to prioritizing marine fish and shellfish populations for conservation. Fish Fish. 2: 328-343.

Pereira, S.M., A. Arias, J. Méndez, A. Insua \& R. Freire. 2009. Isolation of twelve microsatellite markers in the pullet carpet shell Venerupis pullastra (Bivalvia: Veneridae). 2: 201-203.

Powell, W., M. Morgante, J.J. Doyle, J.W. McNicol, S.V. Tingey \& J.A. Rafalski. 1996. Genepool variation in genus Glycine subspecies Soja revealed by polymorphic nuclear and chloroplast microsatellites. Genetics 144: 793-803.

Raymond, M. \& F. Rousset. 1995. Genepop (Version 3.4): population genetics software for exact tests and ecumenicism. J. Hered. 86: 248-249.

Reece, K.S., W.L. Ribeiro, P.M. Gaffney, R.B. Carnegie \& K. Allen Jr. 2004. Microsatellite marker development and analysis in the Eastern Oyster (Crassostrea virginica): Confirmation of null alleles and nonMendelian segregation ratios. J. Hered. 95: 346-352.

Rice, W.R.1989. Analyzing tables of statistical tests. Evolution 41: 223-235.

Slatkin, M. \& N.H. Barton. 1989. A comparison of three indirect methods for estimating average levels of gene flow. Evolution 43: 1349-1368.
Slatkin, M. 1994. Gene flow and population structure, p. 3-17. In L.A. Real (ed.) .Ecological Genetics. Princeton University, Princeton, Nueva Jersey, EEUU.

Sokolov, E.P., I.M. Sokolova \& H.O. Pörtner. 2002. Polymorphic microsatellite DNA markers from the marine gastropod Littorina saxatilis. Mol. Ecol. Notes 2: 27-29.

Tello, C.J.A., L.A. Rodríguez-Gil \& F. Rodríguez-Romero. 2005. Population genetics of the pink snail Strombus gigas in the Yucatan Peninsula: Implications for its management and fishery. Cienc. Mar. 31: 379-386.

Tripp-Valdéz, M., F.J. García de León, S. Ortega-García, D. Lluch-Cota, J. López-Martínez \& P. Cruz. 2010. Population genetic structure of dolphinfish (Coryphaena hippurus) in the Gulf of California, using mirosatellite loci. Fish. Res. 105: 171-1.

Van Oosterhout, C., C. Hutchinson, D.P.M. Wills \& P. Shipley. 2004. Program note Micro-Checker: software for identifying and correcting genotype errors in microsatellite data. Mol. Ecol. Notes 4: 535-538.

Weetman, D., L. Hauser, P.W. Shaw \& M. Bayes. 2005. Microsatellite markers for the whelk Buccinum undatum. Mol. Ecol. Notes 5: 361-362.

Zamora-Bustillos, R., R. Rodríguez-Canul \& F.J. García De León. 2007. Isolation and characterization of eight polymorphic microsatellite markers from pin conch (Strombus gigas). Mol. Ecol. Notes 7: 597-599.

Zi-Niu, Yu, K. Xiao-Yu, G. Tian-Hui, J. Yan-Yan, Z. ZhiMeng \& J. Xian-Shi. 2005. Mitochondrial DNA sequence variation of Japanese Anchovy Engraulis japonicus from the Yellow Sea and East China Sea. Fish. Sci. 71: 299-307. 